\title{
Credibility-Based Biobjective Fuzzy Optimization for Supplier Selection Problem with Disruption
}

\author{
Xuejie Bai ${ }^{1,2}$ \\ ${ }^{1}$ College of Science, Hebei Agricultural University, Baoding, Hebei 071001, China \\ ${ }^{2}$ College of Management, Hebei University, Baoding, Hebei 071002, China \\ Correspondence should be addressed to Xuejie Bai; hebaubxj@163.com
}

Received 14 July 2015; Revised 24 September 2015; Accepted 28 September 2015

Academic Editor: Thomas Hanne

Copyright ( 2015 Xuejie Bai. This is an open access article distributed under the Creative Commons Attribution License, which permits unrestricted use, distribution, and reproduction in any medium, provided the original work is properly cited.

\begin{abstract}
This paper addresses a supplier selection problem in which a buyer procures multiple products from multiple suppliers under disruption risk. The problem is formulated as a new credibility-based biobjective fuzzy optimization model. In the proposed model, cost, capacity, and demand are characterized by fuzzy variables with known possibility distributions. The objectives of our model are to maximize the total quality of purchased products and minimize the expected total cost. Two credibility constraints are used to guarantee that the chance about the supplier capacity and buyer demand can satisfy the predetermined levels. The main concern in solving the optimization model is to calculate the expected value of the objective function and the credibility in the constraints. When the key parameters are mutually independent triangular fuzzy variables, the expected cost objective and credibility constraints can be transformed into their equivalent forms. Taking advantage of the structural characteristics of the equivalent model, the goal programming method is employed to solve the supplier selection model, which can be solved by conventional optimization method. At last, some numerical experiments have been performed to illustrate the effectiveness of the proposed model and solution strategy.
\end{abstract}

\section{Introduction}

Supplier selection is an important component of supply chain management and receives a great deal of attention with the development of economic globalization and competitive pressure. Due to the presence of outside suppliers, supply chains are subject to two sources of risk, routine operational problems and major disruptions [1]. Disruptive events mainly include both natural disasters and man-made disasters, which have caused disastrous damage to various firms or companies in different supply chains. Supplier selection with disruption refers to the fact that suppliers are assigned sequentially so that the buyer may already have one backup when its primary supplier suffers a default due to disruptive risks. The purpose of this paper is to formulate a credibilitybased biobjective optimization model to mitigate disruption risks while addressing concurrently operational risks as well. According to the computational results, the decision maker can obtain a supplier-level-order assignment plan that is used for routine operation and a mitigation scheme that would be prepared when a disruption event occurs.

Some critical parameters in the supplier selection activities are always treated as uncertain variables to meet the practical situations. For instance, if one needs to make a supplier selection plan for the next month, the capacity of each supplier, the demand of the buyer, and the acquisition cost of each product are often estimated by history statistics because of no precise a priori information. The existence of uncertainty has motivated a variety of researches to investigate the supplier selection problem by using stochastic methods. For example, Balcik and Ak [2] addressed the supplier selection problem of a relief organization in preparation for the sudden-onset disasters and developed a stochastic programming model by using a scenario-based approach to represent demand uncertainty. In order to determine the optimal set of suppliers and the aggregate order and its allocation among the suppliers, Federgruen and Yang [3] analyzed a planning model for a single buyer with uncertain 
demand and multiple suppliers with random yields. Guu et al. [4] proposed the optimistic, pessimistic, and combination strategies, three different optimization models with interval coefficients to model the uncertain supplier selection problem. Hosseininasab and Ahmadi [5] introduced a two-phase procedure to determine a supplier portfolio by maximizing the expected value and development of suppliers as well as minimizing their correlated risk. Hu and Kostamis [6] discussed a manufacturer's optimal multiple sourcing strategies when he faced both reliable and unreliable suppliers under supply disruptions. Sawik [7] applied a stochastic mixedinteger programming approach to the joint supplier selection and customer order scheduling in the presence of supply chain disruption risks.

Since the pioneering work of Zadeh [8], fuzzy theory has become a strong tool to cope with possibilistic uncertainty [9-11]. A number of researchers in the literature have employed fuzzy techniques into the supplier selection process. For instance, Amid et al. [12] developed a weighted max-min fuzzy model for supplier selection problem to handle different weights of multiple criteria that were determined by the analytic hierarchy process. Arabzad et al. [13] proposed a supplier selection model where suppliers were evaluated by strengths, weaknesses, opportunities, and threats analysis in the first phase and order amounts were determined through fuzzy TOPSIS method in the second phase. Arikan [14] formulated multiple objectives linear programming problem with fuzzy demand level and fuzzy aspiration levels of objectives for selecting appropriate suppliers and solved it by a novel interactive solution procedure. Azadnia et al. [15] designed an integrated approach of rulebased weighted fuzzy method, fuzzy analytical hierarchy process, and multiobjective mathematical programming for sustainable supplier selection problem and illustrated its applicability by a case study of packaging films in food industry. Guneri et al. [16] presented an integrated fuzzy and linear programming approach for supplier selection problem. Pan et al. [17] proposed a fuzzy multiobjective model for supplier selection under considering stochastic demand and transformed the original model into a weighted max-min deterministic linear/nonlinear one to find the optimal solution. For a thorough coverage of supplier selection problem, the interested reader may refer to Chai et al. [18] and references therein.

Compared to some related studies on supplier selection problem, the main contributions of the current research include the following several aspects. Firstly, this paper formulates a novel class of biobjective supplier selection model on the basis of fuzzy optimization theory. In the literature, a variety of fuzzy supplier selection models employ the multiple criteria decision making techniques. Our adopted optimization method distinguishes this study from other fuzzy supplier selection models. Secondly, our established model considers the impact of fuzzy cost, capacity, and demand parameters on the optimal supplier-level-order strategy. Most work in this field provided the supplier selection decisions based on a limited set of scenarios, which may be frustrating once there was not enough historical data. Thirdly, the basic properties of proposed model are discussed in case that the uncertain parameters are mutually independent triangular fuzzy variables. After that a solution method integrated with goal programming is designed to turn the original fuzzy biobjective model into the equivalent deterministic single objective one. Finally, from the application point of view, a supplier selection numerical example is presented. The computational results show the effectiveness and superiority of established biobjective fuzzy optimization model and designed solution procedure.

The rest of this paper is organized as follows. Section 2 formulates a new class of fuzzy supplier selection model. Section 3 focuses on the deterministic equivalent expressions to the expected objective and credibility constraints. In Section 4, one solution approach, preemptive goal programming method, is designed. To apply the proposed model to a decision making problem, Section 5 presents a selection example with one buyer, three products, and five suppliers. Finally, Section 6 concludes the paper and gives the future work.

\section{Fuzzy Biobjective Model for Supplier Selection Problem}

This section aims to use fuzzy optimization methodology to formulate a credibility-based supplier selection model. In the supplier selection decision making process, there are multiple suppliers and multiple products for a single buyer, which is faced by most purchasing organizations. In order to hedge against operational risks as well as mitigate against disruptions, all the suppliers are separated as primary suppliers and backup ones. Primary suppliers are used to sign long-term procurement contracts for routine operation, while backups will not make short-term trade-offs with the buyer unless disruptive events erupt on one or more primary suppliers. Snyder and Daskin [19] introduced the concept of level- $r$ facility to deal with sequential assignment of facilities to customers in the facility location problem. Motivated by the above-mentioned research, in our model the primary suppliers are assigned to level-1 suppliers for the given product. The remaining backup ones are then sequentially assigned to level-2 supplier, level-3 supplier, and so on. Once a disruption occurs, the primary suppliers fail and the backup ones are used to provide order fulfillment. The model accommodates a mitigation plan through assigning suppliers at consecutive levels and an optimal order decision. The assumptions, notations used in the formulation, and the formulation process are explained as follows.

\section{Assumptions}

(A1) A joint replenishment policy is adopted for all products.

(A2) The supplier will afford products with the same quality level whenever he is a primary supplier or a backup.

(A3) The higher the level, the higher the variable cost together with longer lead time but lower fixed cost, which makes sense that the buyer will not use backup supplier unless one primary fails. 
Constraint Conditions. (I) Constraint (1) ensures that each product is allowed to have at most $m$ level-1 suppliers; that is,

$$
\sum_{s \in S} x_{1 s p} \leq m, \quad \forall p \in P
$$

(II) Constraint (2) imposes that a single product can have only one backup supplier for each level; that is,

$$
\sum_{s \in S} x_{r s p}=1, \quad \forall p \in P, r=2,3, \ldots, n-m+1
$$

(III) Constraint (3) prohibits assigning one supplier to more than one level for the same product, which should be represented by

$$
\sum_{r \in R} x_{r s p}=1, \quad \forall s \in S, p \in P
$$

The aforementioned three equations (1)-(3) are the basic ideas of caving up suppliers. In our paper, the replacement scheme follows that the backup supplier with one level below will replace the failed supplier in case of a default at a primary supplier. When two primary ones are exposed to disruptive risk, both level-2 and level-3 suppliers will be employed to afford indents. Similarly, when $m^{\prime}\left(m^{\prime} \leq m\right)$ primary ones suffer "fail," level-2 to level- $m$ ' backup suppliers, if existing, will receive tasks until all suppliers have been employed.

(IV) Constraint (4) ensures that the total lead time does not exceed the acceptable maximal lead time:

$$
\sum_{s \in S} \sum_{p \in P} L_{1 s p} y_{1 s p}+\sum_{s \in S} \sum_{p \in P} \sum_{r=2}^{n-m+1} L_{r s p} x_{r s p} \leq L
$$

(V) Constraint (5) imposes that the corresponding order sizes should be restricted to its capacity once supplier $s$ has been a primary dealer for product $p$; that is,

$$
\operatorname{Cr}\left\{y_{1 s p} \leq \eta_{s p} x_{1 s p}\right\} \geq \alpha_{s p}, \quad \forall s \in S, p \in P,
$$

where the parameter $\alpha_{s p}$ represents capacity level for supplier $s$.
(VI) Constraint (6) ensures that the order quantities received by all for some product could meet the buyer demand; that is,

$$
\operatorname{Cr}\left\{\sum_{s \in S} y_{1 s p} \geq \zeta_{p}\right\} \geq \beta_{p}, \quad \forall p \in P,
$$

where the parameter $\beta_{p}$ represents service level for product p.

(VII) Constraints (7) guarantee that the binary and nonnegativity restrictions on the decision variables hold:

$$
\begin{aligned}
& x_{r s p} \in\{0,1\}, \quad \forall s \in S, p \in P, \\
& y_{1 s p} \geq 0, \quad \forall s \in S, p \in P .
\end{aligned}
$$

Objective Functions. The first objective function is to maximize the total quality of purchased products. The total quality is divided into two parts: primary suppliers' quality and an opportunity quality; that is,

$$
\max \sum_{s \in S} \sum_{p \in P} Q_{s p} y_{1 s p}+\sum_{s \in S} \sum_{p \in P} \sum_{r=2}^{n-m+1} Q_{s p} x_{r s p},
$$

where $Q_{s p}$ has nothing with priority levels.

The second objective function is to minimize the total cost. The total cost is comprised of the fixed cost and variable cost. The fixed cost that the buyer spends in signing a contact with every supplier is defined as $\sum_{s \in S} \sum_{p \in P} \sum_{r=1}^{n-m+1} F_{r s p} x_{r s p}$. The variable cost including the purchasing, transportation, and holding cost from primary suppliers can be represented by $\sum_{s \in S} \sum_{p \in P} \xi_{1 s p} y_{1 s p}$, while the one from backup suppliers, also called an opportunity cost due to not using backup suppliers at the beginning, can be represented by $\sum_{s \in S} \sum_{p \in P} \sum_{r=2}^{n-m+1} \xi_{r s p} x_{r s p}$. The expected total cost can be minimized; that is,

$$
\begin{aligned}
& \min \mathrm{E}\left\{\sum_{s \in S} \sum_{p \in P} \sum_{r \in R} F_{r s p} x_{r s p}+\sum_{s \in S} \sum_{p \in P} \xi_{1 s p} y_{1 s p}\right. \\
& \left.+\sum_{s \in S} \sum_{p \in P} \sum_{r=2}^{n-m+1} \xi_{r s p} x_{r s p}\right\} .
\end{aligned}
$$

Based on the above analysis, a credibility-based biobjective fuzzy supplier selection optimization model is formally built as follows:

$$
\begin{aligned}
& \max \sum_{s \in S} \sum_{p \in P} Q_{s p} y_{1 s p}+\sum_{s \in S} \sum_{p \in P} \sum_{r=2}^{n-m+1} Q_{s p} x_{r s p} \\
& \min \quad \mathrm{E}\left\{\sum_{s \in S} \sum_{p \in P} \sum_{r \in R} F_{r s p} x_{r s p}+\sum_{s \in S} \sum_{p \in P} \xi_{1 s p} y_{1 s p}+\sum_{s \in S} \sum_{p \in P} \sum_{r=2}^{n-m+1} \xi_{r s p} x_{r s p}\right\}
\end{aligned}
$$




$$
\begin{array}{ll}
\text { s.t. } & \sum_{s \in S} x_{1 s p} \leq m, \quad \forall p \in P, \\
& \sum_{s \in S} x_{r s p}=1, \quad \forall p \in P, r=2,3, \ldots, n-m+1, \\
& \sum_{r \in R} x_{r s p}=1, \quad \forall s \in S, p \in P, \\
& \sum_{s \in S} \sum_{p \in P} L_{1 s p} y_{1 s p}+\sum_{s \in S} \sum_{p \in P} \sum_{r=2}^{n-m+1} L_{r s p} x_{r s p} \leq L, \\
& \operatorname{Cr}\left\{y_{1 s p} \leq \eta_{s p} x_{1 s p}\right\} \geq \alpha_{s p}, \quad \forall s \in S, p \in P, \\
& \operatorname{Cr}\left\{\sum_{s \in S} y_{1 s p} \geq \zeta_{p}\right\} \geq \beta_{p}, \quad \forall p \in P, \\
& x_{r s p} \in\{0,1\}, \quad \forall r \in R, s \in S, p \in P, \\
& y_{1 s p} \geq 0, \quad \forall s \in S, p \in P .
\end{array}
$$

Model (10) is a biobjective mixed-integer programming with the expectation objective and credibility constraints. The general solution methods require conversion of expected value and credibility to their respective deterministic equivalent forms. Usually, this transformation is hard to perform and only successful for some special cases. In the subsequent section, the equivalent formulation of model (10) will be discussed.

\section{Model Analysis}

To solve model (10), the key challenges are to calculate the expected value objective in terms of the total cost and check the credibility constraints with respect to supplier capacity and service level. The main purpose of this section will consider some special cases that all fuzzy variables are triangularly distributed and concern the equivalent expressions.

Assume $\xi_{r s p}, \eta_{s p}$, and $\zeta_{p}$ are mutually independent triangular fuzzy variables such that their elements are defined by

$$
\begin{aligned}
\xi_{r s p} & =\left(\xi_{r s p}^{r_{1}}, \xi_{r s p}^{r_{2}}, \xi_{r s p}^{r_{3}}\right), \\
\eta_{s p} & =\left(\eta_{s p}^{r_{1}}, \eta_{s p}^{r_{2}}, \eta_{s p}^{r_{3}}\right), \\
\zeta_{p} & =\left(\zeta_{p}^{r_{1}}, \zeta_{p}^{r_{2}}, \zeta_{p}^{r_{3}}\right) .
\end{aligned}
$$

The first theorem considers the analytical expression of total cost objective; that is,

$$
\begin{gathered}
\mathrm{E}\left\{\sum_{s \in S} \sum_{p \in P} \sum_{r \in R} F_{r s p} x_{r s p}+\sum_{s \in S} \sum_{p \in P} \xi_{1 s p} y_{1 s p}\right. \\
\left.+\sum_{s \in S} \sum_{p \in P} \sum_{r=2}^{n-m+1} \xi_{r s p} x_{r s p}\right\} .
\end{gathered}
$$

Theorem 1. Consider total cost objective in supplier selection model (10). Let the fuzzy cost $\xi_{r s p}=\left(\xi_{r s p}^{r_{1}}, \xi_{r s p}^{r_{2}}, \xi_{r s p}^{r_{3}}\right)$ for unit product $p$ from its level-r supplier $s$ be a triangular fuzzy variable. Suppose $\xi_{111}, \xi_{112}, \ldots, \xi_{n-m+1|S||P|}$ are mutually independent; then total cost objective is equivalent to

$$
\begin{aligned}
& \sum_{s \in S} \sum_{p \in P} \sum_{r \in R} F_{r s p} x_{r s p}+\sum_{s \in S} \sum_{p \in P} \frac{\xi_{1 s p}^{r_{1}}+2 \xi_{1 s p}^{r_{2}}+\xi_{1 s p}^{r_{3}}}{4} y_{1 s p} \\
& +\sum_{s \in S} \sum_{p \in P} \sum_{r=2}^{n-m+1} \frac{\xi_{r s p}^{r_{1}}+2 \xi_{r s p}^{r_{2}}+\xi_{r s p}^{r_{3}}}{4} x_{r s p} .
\end{aligned}
$$

Proof. Suppose $\xi_{r s p}$ is a triangular fuzzy variable. Based on the definition of mathematical expectation [9], it follows that the expected value of $\xi_{r s p}$ is

$$
\mathrm{E}\left[\xi_{r s p}\right]=\frac{\xi_{r s p}^{r_{1}}+2 \xi_{r s p}^{r_{2}}+\xi_{r s p}^{r_{3}}}{4}
$$

Since $\xi_{111}, \xi_{112}, \ldots, \xi_{n-m+1|S||P|}$ are mutually independent, due to the linearity of expectation, we have

$$
\begin{aligned}
& \mathrm{E}\left\{\sum_{s \in S} \sum_{p \in P} \sum_{r \in R} F_{r s p} x_{r s p}+\sum_{s \in S} \sum_{p \in P} \xi_{1 s p} y_{1 s p}\right. \\
& \left.+\sum_{s \in S} \sum_{p \in P} \sum_{r=2}^{n-m+1} \xi_{r s p} x_{r s p}\right\}=\sum_{s \in S} \sum_{p \in P} \sum_{r \in R} F_{r s p} x_{r s p} \\
& +\mathrm{E}\left\{\sum_{s \in S} \sum_{p \in P} \xi_{1 s p} y_{1 s p}+\sum_{s \in S} \sum_{p \in P} \sum_{r=2}^{n-m+1} \xi_{r s p} x_{r s p}\right\}
\end{aligned}
$$




$$
\begin{aligned}
& =\sum_{s \in S} \sum_{p \in P} \sum_{r \in R} F_{r s p} x_{r s p}+\sum_{s \in S} \sum_{p \in P} \mathrm{E}\left[\xi_{1 s p}\right] y_{1 s p} \\
& +\sum_{s \in S} \sum_{p \in P} \sum_{r=2}^{n-m+1} \mathrm{E}\left[\xi_{r s p}\right] x_{r s p} .
\end{aligned}
$$

Therefore, total cost objective is equivalent to

$$
\begin{aligned}
& \sum_{s \in S} \sum_{p \in P} \sum_{r \in R} F_{r s p} x_{r s p}+\sum_{s \in S} \sum_{p \in P} \frac{\xi_{1 s p}^{r_{1}}+2 \xi_{1 s p}^{r_{2}}+\xi_{1 s p}^{r_{3}}}{4} y_{1 s p} \\
& +\sum_{s \in S} \sum_{p \in P} \sum_{r=2}^{n-m+1} \frac{\xi_{r s p}^{r_{1}}+2 \xi_{r s p}^{r_{2}}+\xi_{r s p}^{r_{3}}}{4} x_{r s p} .
\end{aligned}
$$

The proof of theorem is complete.

The following theorem establishes the analytical expressions of supplier capacity constraint; that is,

$$
\operatorname{Cr}\left\{y_{1 s p} \leq \eta_{s p} x_{1 s p}\right\} \geq \alpha_{s p}, \quad \forall s \in S, p \in P .
$$

Theorem 2. Consider supplier capacity constraint in supplier selection model (10). Let the fuzzy capacity $\eta_{s p}=\left(\eta_{s p}^{r_{1}}, \eta_{s p}^{r_{2}}, \eta_{s p}^{r_{3}}\right)$ at supplier s for product $p$ be a triangular fuzzy variable.

(i) If $\alpha_{s p} \in(0,0.5)$, then supplier capacity constraint is equivalent to

$$
y_{1 s p} \leq\left[\eta_{s p}^{r_{3}}-2 \alpha_{s p}\left(\eta_{s p}^{r_{3}}-\eta_{s p}^{r_{2}}\right)\right] x_{1 s p}, \quad \forall s \in S, p \in P .
$$

(ii) If $\alpha_{s p} \in[0.5,1]$, then supplier capacity constraint is equivalent to

$$
y_{1 s p} \leq\left[\eta_{s p}^{r_{1}}+2\left(1-\alpha_{s p}\right)\left(\eta_{s p}^{r_{2}}-\eta_{s p}^{r_{1}}\right)\right] x_{1 s p}
$$

$\forall s \in S, p \in P$.

Proof. We only prove assertion (ii), and assertion (i) can be proved similarly.

Suppose $\eta_{s p}$ is a triangular fuzzy variable. The possibility distribution of $\eta_{s p}$ is

$$
\mu_{\eta_{s p}}(x)= \begin{cases}\frac{x-\eta_{s p}^{r_{1}}}{\eta_{s p}^{r_{2}}-\eta_{s p}^{r_{1}}}, & \text { if } x \in\left[\eta_{s p}^{r_{1}}, \eta_{s p}^{r_{2}}\right), \\ \frac{\eta_{s p}^{r_{3}}-x}{\eta_{s p}^{r_{3}}-\eta_{s p}^{r_{2}}}, & \text { if } x \in\left[\eta_{s p}^{r_{2}}, \eta_{s p}^{r_{3}}\right] .\end{cases}
$$

Denote $\eta=\eta_{s p} x_{1 s p}$. If $\alpha_{s p} \geq 0.5$, according to credibility measure, then we have

$$
\begin{aligned}
\operatorname{Cr}\left\{y_{1 s p} \leq \eta_{s p} x_{1 s p}\right\} & =\operatorname{Cr}\{\eta \geq t\} \\
& =\frac{1}{2}\left(1+\sup _{x \geq t} \mu_{\eta}(x)-\sup _{x<t} \mu_{\eta}(x)\right) \\
& =\frac{1}{2}\left(2-\sup _{x<t} \mu_{\eta}(x)\right)
\end{aligned}
$$

where $t=y_{1 s p}$. Thus, $\operatorname{Cr}\left\{y_{1 s p} \leq \eta_{s p} x_{1 s p}\right\} \geq \alpha_{s p}$ is equivalent to $\sup _{x<t} \mu_{\eta}(x) \leq 2-2 \alpha_{s p}$.

If we denote

$$
\eta_{\text {inf }}(\alpha)=\sup \left\{t \mid \sup _{x<t} \mu_{\eta}(x) \leq \alpha\right\}
$$

for $\alpha \in(0,1]$, then we have $\eta_{\text {inf }}\left(2-2 \alpha_{s p}\right) \geq t$. Together with $x_{1 s p} \geq 0$, it follows from the property of fractile function that

$$
\begin{aligned}
\eta_{\text {inf }}\left(2-2 \alpha_{s p}\right) & =\left(\eta_{s p} x_{1 s p}\right)_{\text {inf }}\left(2-2 \alpha_{s p}\right) \\
& =\eta_{s p \text {,inf }}\left(2-2 \alpha_{s p}\right) x_{1 s p} \geq y_{1 s p} .
\end{aligned}
$$

If $\alpha_{s p} \geq 0.5$, then $\eta_{s p \text {,inf }}\left(2-2 \alpha_{s p}\right)$ is the solution of the following equation:

$$
\frac{x-\eta_{s p}^{r_{1}}}{\eta_{s p}^{r_{2}}-\eta_{s p}^{r_{1}}}-\left(2-2 \alpha_{s p}\right)=0 .
$$

Solving the above equation, we have

$$
\eta_{s p, \text { inf }}\left(2-2 \alpha_{s p}\right)=\eta_{s p}^{r_{1}}+2\left(1-\alpha_{s p}\right)\left(\eta_{s p}^{r_{2}}-\eta_{s p}^{r_{1}}\right) .
$$

Therefore, supplier capacity constraint is equivalent to

$$
y_{1 s p} \leq\left[\eta_{s p}^{r_{1}}+2\left(1-\alpha_{s p}\right)\left(\eta_{s p}^{r_{2}}-\eta_{s p}^{r_{1}}\right)\right] x_{1 s p} .
$$

The proof of theorem is complete.

The last theorem constructs the analytical expressions of service level constraint; that is,

$$
\operatorname{Cr}\left\{\sum_{s \in S} y_{1 s p} \geq \zeta_{p}\right\} \geq \beta_{p}, \quad \forall p \in P .
$$

Theorem 3. Consider service level constraint in supplier selection model (10). Let the fuzzy demand $\zeta_{p}=\left(\zeta_{p}^{r_{1}}, \zeta_{p}^{r_{2}}, \zeta_{p}^{r_{3}}\right)$ for product $p$ be a triangular fuzzy variable.

(i) If $\beta_{p} \in(0,0.5)$, then service level constraint is equivalent to

$$
\sum_{s \in S} y_{1 s p} \geq \zeta_{p}^{r_{1}}+2 \beta_{p}\left(\zeta_{p}^{r_{2}}-\zeta_{p}^{r_{1}}\right), \quad \forall p \in P
$$

(ii) If $\beta_{p} \in[0.5,1]$, then service level constraint is equivalent to

$$
\sum_{s \in S} y_{1 s p} \geq \zeta_{p}^{r_{3}}-2\left(1-\beta_{p}\right)\left(\zeta_{p}^{r_{3}}-\zeta_{p}^{r_{2}}\right), \quad \forall p \in P
$$

Proof. We only prove assertion (i), and assertion (ii) can be proved similarly.

Suppose $\zeta_{p}$ is a triangular fuzzy variable. The possibility distribution of $\zeta_{p}$ is

$$
\mu_{\zeta_{p}}(x)= \begin{cases}\frac{x-\zeta_{p}^{r_{1}}}{\zeta_{p}^{r_{2}}-\zeta_{p}^{r_{1}}}, & \text { if } x \in\left[\zeta_{p}^{r_{1}}, \zeta_{p}^{r_{2}}\right), \\ \frac{\zeta_{p}^{r_{3}}-x}{\zeta_{p}^{r_{3}}-\zeta_{p}^{r_{2}}}, & \text { if } x \in\left[\zeta_{p}^{r_{2}}, \zeta_{p}^{r_{3}}\right] .\end{cases}
$$


If $\beta_{p}<0.5$, then we have

$$
\begin{aligned}
\operatorname{Cr} & \left\{\sum_{s \in S} y_{1 s p} \geq \zeta_{p}\right\}=\operatorname{Cr}\left\{\zeta_{p} \leq \sum_{s \in S} y_{1 s p}\right\} \\
& =\frac{1}{2}\left(1+\sup _{x \leq r} \mu_{\zeta_{p}}(x)-\sup _{x>r} \mu_{\zeta_{p}}(x)\right) \\
& =\frac{1}{2} \sup _{x \leq r} \mu_{\zeta_{p}}(x)
\end{aligned}
$$

where $r=\sum_{s \in S} y_{1 s p}$. Thus, $\operatorname{Cr}\left\{\sum_{s \in S} y_{1 s p} \geq \zeta_{p}\right\} \geq \beta_{p}$ is equivalent to $\sup _{x \leq r} \mu_{\zeta_{p}}(x) \geq 2 \beta_{p}$.

If we denote

$$
\zeta_{p, \text { inf }}(\beta)=\inf \left\{r \mid \sup _{x \leq r} \mu_{\zeta_{p}}(x) \geq \beta\right\}
$$

for $\beta \in(0,1]$, then we have $\zeta_{p \text {,inf }}\left(2 \beta_{p}\right) \leq r$.

If $\beta_{p}<0.5$, then $\zeta_{p \text {,inf }}\left(2 \beta_{p}\right)$ is the solution of the following equation:

$$
\frac{x-\zeta_{p}^{r_{1}}}{\zeta_{p}^{r_{2}}-\zeta_{p}^{r_{1}}}-2 \beta_{p}=0
$$

Solving the above equation, we have

$$
\zeta_{p, \text { inf }}\left(2 \beta_{p}\right)=\zeta_{p}^{r_{1}}+2 \beta_{p}\left(\zeta_{p}^{r_{2}}-\zeta_{p}^{r_{1}}\right)
$$

Therefore, service level constraint is equivalent to

$$
\sum_{s \in S} y_{1 s p} \geq \zeta_{p}^{r_{1}}+2 \beta_{p}\left(\zeta_{p}^{r_{2}}-\zeta_{p}^{r_{1}}\right), \quad \forall p \in P .
$$

The proof of theorem is complete.

\section{Solution Method}

The deterministic equivalent formulation of original supplier selection model is a multiobjective optimization problem. There are a great number of solution methods such as $\epsilon$ constraint, weighted sum, lexicographic min-max method, and intelligent algorithm that have been developed for solving such problems. For example, Wang et al. [20] designed a multiobjective algorithm that integrated the guided genetic algorithm and the nondominated sorting genetic algorithm II to solve the problems of supplier selection, assembly sequence planning, and assembly line balancing. The interested readers can refer to Chinchuluun and Pardalos [21], Collette and Siarry [22], and Deb [23] for details on other solution approaches and applications of multiobjective optimization.

When planning the supplier selection system, the goals considered in the original model have economical and quality implications. Multiple goals with appropriate priority structure must be taken into consideration, so it is the best choice to employ the goal programming method to convert a multiobjective optimization model into a single objective one. The goal programming method was introduced by Charnes et al. [24], extensively discussed by Charnes and Cooper [25], and further refined by the work of Ijiri [26] and Lee [27]. The solution process for our mixed-integer goal programming is described as follows.

Firstly, determine the preemptive level for every objective according to the decision maker's preference.

For the quality objective function, the target value $G_{1}$ is given by senior decision maker who makes the decision relying on previous information or personal preference. When the target value is fixed, additional variables $d_{1}^{+}$ and $d_{1}^{-}$can be obtained, serving as positive and negative deviation variables. Here, $d_{1}^{+}$represents the segment of the first objective exceeding its target value, and $d_{1}^{-}$denotes the segment of the first objective less than its target value. The soft constraint is chalked up well by involving the negative and positive deviation variables together with the target value into the first objective. Note that the quality objective is required to find the maximum value. Therefore, the smaller the $d_{1}^{-}$is, the best the objective is:

$$
\begin{array}{ll}
\min & d_{1}^{-} \\
\text {s.t. } & \sum_{s \in S} \sum_{p \in P} Q_{s p} y_{1 s p}+\sum_{s \in S} \sum_{p \in P} \sum_{r=2}^{n-m+1} Q_{s p} x_{r s p}+d_{1}^{-}-d_{1}^{+} \\
& =G_{1} .
\end{array}
$$

Similarly, the total cost objective function can be written as follows:

$$
\begin{array}{ll}
\min & d_{2}^{+} \\
\text {s.t. } \quad \sum_{s \in S} \sum_{p \in P} \sum_{r \in R} F_{r s p} x_{r s p} \\
\\
+\sum_{s \in S} \sum_{p \in P} \frac{\xi_{1 s p}^{r_{1}}+2 \xi_{1 s p}^{r_{2}}+\xi_{1 s p}^{r_{3}}}{4} y_{1 s p} \\
\quad+\sum_{s \in S} \sum_{p \in P} \sum_{r=2}^{n-m+1} \frac{\xi_{r s p}^{r_{1}}+2 \xi_{r s p}^{r_{2}}+\xi_{r s p}^{r_{3}}}{4} x_{r s p}+d_{2}^{-}-d_{2}^{+} \\
=G_{2} .
\end{array}
$$

The decision maker assigns these two objectives with preemptive levels $P_{1}$ and $P_{2}$, complying with $P_{1} \gg P_{2}$. That is to say, when formulating the criteria function, it is requested to order the unfavorable deviations into a number of preemptive levels, with the minimization of a deviation in a higher preemptive level being infinitely more important than any deviations in lower preemptive levels. Assume the cost objective is prior to the quality objective. The criteria function minimizes the sum of the deviations from the target values specified in the soft constraints appended above:

$$
\min P_{1} d_{1}^{-}+P_{2} d_{2}^{+} \text {. }
$$

Secondly, specify the domain for capacity parameter $\alpha_{s p}$ and service level $\beta_{p}$.

On the basis of Theorem 2, if $\alpha_{s p} \geq 0.5$ and $\eta_{s p}$ is triangularly distributed, then the supplier capacity constraint

$$
\operatorname{Cr}\left\{y_{1 s p} \leq \eta_{s p} x_{1 s p}\right\} \geq \alpha_{s p}, \quad \forall p \in P,
$$


has the following equivalent expression:

$$
\begin{aligned}
y_{1 s p} \leq\left[\eta_{s p}^{r_{1}}+2\left(1-\alpha_{s p}\right)\left(\eta_{s p}^{r_{2}}-\eta_{s p}^{r_{1}}\right)\right] & x_{1 s p} \\
& \\
& \forall s \in S, p \in P .
\end{aligned}
$$

According to Theorem 3 , if $\beta_{p} \geq 0.5$ and $\zeta_{p}$ is a triangular fuzzy variable, then the service level constraint

$$
\operatorname{Cr}\left\{\sum_{s \in S} y_{1 s p} \geq \zeta_{p}\right\} \geq \beta_{p}, \quad \forall p \in P,
$$

is equivalent to the following inequality:

$$
\sum_{s \in S} y_{1 s p} \geq \zeta_{p}^{r_{3}}-2\left(1-\beta_{p}\right)\left(\zeta_{p}^{r_{3}}-\zeta_{p}^{r_{2}}\right), \quad \forall p \in P .
$$

Thirdly, solve the deterministic mixed-integer goal programming model.

The preemptive goal programming formulation of the supplier selection model is given as follows:

\begin{tabular}{|c|c|c|c|c|c|}
\hline \multirow{2}{*}{ Product } & \multicolumn{5}{|c|}{ Quality } \\
\hline & Supplier 1 & Supplier 2 & Supplier 3 & Supplier 4 & Supplier 5 \\
\hline 1 & 0.95 & 0.95 & 0.9 & 0.9 & 0.9 \\
\hline 2 & 0.95 & 0.97 & 0.9 & 0.93 & 0.92 \\
\hline 3 & 0.93 & 0.99 & 0.9 & 0.9 & 0.97 \\
\hline
\end{tabular}

$$
\begin{aligned}
& \min P_{1} d_{1}^{-}+P_{2} d_{2}^{+} \\
& \text {s.t. } \sum_{s \in S} \sum_{p \in P} Q_{s p} y_{1 s p}+\sum_{s \in S} \sum_{p \in P} \sum_{r=2}^{n-m+1} Q_{s p} x_{r s p}+d_{1}^{-}-d_{1}^{+} \\
& =G_{1} \text {, } \\
& \sum_{s \in S} \sum_{p \in P} \sum_{r \in R} F_{r s p} x_{r s p} \\
& +\sum_{s \in S} \sum_{p \in P} \frac{\xi_{1 s p}^{r_{1}}+2 \xi_{1 s p}^{r_{2}}+\xi_{1 s p}^{r_{3}}}{4} y_{1 s p} \\
& +\sum_{s \in S} \sum_{p \in P} \sum_{r=2}^{n-m+1} \frac{\xi_{r s p}^{r_{1}}+2 \xi_{r s p}^{r_{2}}+\xi_{r s p}^{r_{3}}}{4} x_{r s p}+d_{2}^{-}-d_{2}^{+} \\
& =G_{2} \text {, } \\
& \sum_{s \in S} x_{1 s p} \leq m, \quad \forall p \in P, \\
& \sum_{s \in S} x_{r s p}=1, \quad \forall p \in P, r=2,3, \ldots, n-m+1, \\
& \sum_{r \in R} x_{r s p}=1, \quad \forall s \in S, p \in P, \\
& \sum_{s \in S} \sum_{p \in P} L_{1 s p} y_{1 s p}+\sum_{s \in S} \sum_{p \in P} \sum_{r=2}^{n-m+1} L_{r s p} x_{r s p} \leq L, \\
& y_{1 s p} \leq\left[\eta_{s p}^{r_{1}}+2\left(1-\alpha_{s p}\right)\left(\eta_{s p}^{r_{2}}-\eta_{s p}^{r_{1}}\right)\right] x_{1 s p}, \\
& \forall s \in S, p \in P, \\
& \sum_{s \in S} y_{1 s p} \geq \zeta_{p}^{r_{3}}-2\left(1-\beta_{p}\right)\left(\zeta_{p}^{r_{3}}-\zeta_{p}^{r_{2}}\right)
\end{aligned}
$$

$\forall p \in P$,
TABLE 1: Quality data for supplier selection problem.

$$
\forall r \in R, s \in S, p \in P \text {. }
$$

Model (43) is a mixed-integer parametric programming. It can be solved by using conventional optimization algorithms when the parameters vary in their domains. For example, given the parameters $\alpha_{s p}$ and $\beta_{p}$, we can make use of the branch-and-bound method to solve it. It is known that the LINGO is a state-of-the-art commercial tool including the branch-and-bound IP code. In the next section, the effectiveness of the goal programming method can be demonstrated via numerical experiments.

However, the size of the supplier selection problem may be a serious disadvantage. As the real instance increases the number of suppliers or products, direct solution using standard software may become much less attractive due to the memory requirements. It is of special value in developing a more efficient customized algorithm designed to exploit the structure of the proposed model for large-scale instances, which is a significant avenue for further work.

\section{Numerical Example}

This section reports the computational results for the proposed model and solution approach.

5.1. Problem Description. Suppose that a single buyer needs to purchase three kinds of products. He has identified five potential suppliers and has classified them into three assignment levels where there are three primary suppliers. The data in respect of product quality, unit fixed cost, and lead time have been provided by Tables 1,2 , and 3 . The data in terms of variable cost, supplier capacity, and buyer demand are observed from the historical data where there exist many fluctuations. They have been represented as fuzzy numbers with triangular distributions in Tables 4 and 5. The demand for three products is $(140,160,190),(220,250,280)$, and $(200,230,240)$, respectively. The buyer wants to select the primary and backup suppliers and decide the order amounts among selected suppliers so as to maximize the total quality of products and minimize the total cost of the supplier selection.

5.2. Computational Results and Discussions. When the model parameters $\alpha_{s p}, \beta_{p}, G_{1}, G_{2}$, and $L$ are set to $0.95,0.95$, 700,6000 , and 2800 , respectively, we obtain the maximal 
TABLE 2: Fixed cost data for supplier selection problem.

\begin{tabular}{|c|c|c|c|c|c|c|c|c|c|}
\hline \multirow{2}{*}{ Supplier } & \multicolumn{3}{|c|}{ Product 1} & \multicolumn{3}{|c|}{ Product 2} & \multicolumn{3}{|c|}{ Product 3} \\
\hline & Level-1 & Level-2 & Level-3 & Level-1 & Level-2 & Level-3 & Level-1 & Level-2 & Level-3 \\
\hline 1 & 100 & 95 & 90 & 75 & 65 & 60 & 56 & 50 & 45 \\
\hline 2 & 200 & 160 & 150 & 150 & 130 & 120 & 113 & 108 & 103 \\
\hline 3 & 150 & 130 & 120 & 113 & 105 & 95 & 84 & 70 & 65 \\
\hline 4 & 150 & 135 & 110 & 120 & 108 & 100 & 90 & 85 & 70 \\
\hline 5 & 120 & 112 & 102 & 90 & 80 & 60 & 66 & 60 & 56 \\
\hline
\end{tabular}

TABLE 3: Lead time data for supplier selection problem.

\begin{tabular}{|c|c|c|c|c|c|c|c|c|c|}
\hline \multirow{2}{*}{ Supplier } & \multicolumn{3}{|c|}{ Product 1} & \multicolumn{3}{|c|}{ Product 2} & \multicolumn{3}{|c|}{ Product 3} \\
\hline & Level-1 & Level-2 & Level-3 & Level-1 & Level-2 & Level-3 & Level-1 & Level-2 & Level-3 \\
\hline 1 & 10 & 10.5 & 10.8 & 9 & 9.5 & 9.9 & 1 & 1.1 & 1.2 \\
\hline 2 & 5 & 5.3 & 5.5 & 2 & 2.1 & 2.2 & 8 & 8.4 & 8.8 \\
\hline 3 & 8 & 8.4 & 8.6 & 3 & 3.2 & 3.3 & 6 & 6.2 & 6.4 \\
\hline 4 & 3 & 3.1 & 3.3 & 4 & 4.2 & 4.4 & 6 & 6.3 & 6.6 \\
\hline 5 & 8 & 8.3 & 8.8 & 2 & 2.1 & 2.3 & 4 & 4.2 & 4.5 \\
\hline
\end{tabular}

TABLE 4: Variable cost data for supplier selection problem.

\begin{tabular}{|c|c|c|c|c|}
\hline \multirow{2}{*}{ Supplier } & \multirow{2}{*}{ Product } & \multicolumn{3}{|c|}{ Variable cost } \\
\hline & & Level-1 & Level-2 & Level-3 \\
\hline \multirow{3}{*}{1} & 1 & $(9.5,10,10.7)$ & $(11,11.5,12)$ & $(12,12.5,13)$ \\
\hline & 2 & $(6,7,7.3)$ & $(7.4,7.5,7.9)$ & $(8,8.3,8.5)$ \\
\hline & 3 & $(6,7,7.3)$ & $(10.5,10.6,11)$ & $(11.2,11.3,11.6)$ \\
\hline \multirow{3}{*}{2} & 1 & $(9,10,10.5)$ & $(10.5,10.75,11)$ & $(11.2,11.5,11.8)$ \\
\hline & 2 & $(5.5,6,6.2)$ & $(6.3,6.4,6.5)$ & $(6.6,6.8,7)$ \\
\hline & 3 & $(5,6,6.3)$ & $(6.4,6.5,6.7)$ & $(6.8,6.9,8)$ \\
\hline \multirow{3}{*}{3} & 1 & $(5.8,6,6.35)$ & $(6.4,6.5,6.8)$ & $(6.85,7,7.5)$ \\
\hline & 2 & $(4.2,5,5.2)$ & $(5.25,5.4,5.6)$ & $(5.65,5.9,6)$ \\
\hline & 3 & $(2.4,3,3.2)$ & $(3.25,3.3,3.5)$ & $(3.5,3.6,4)$ \\
\hline \multirow{3}{*}{4} & 1 & $(9,10,10.2)$ & $(10.4,10.8,11)$ & $(11,11.5,12)$ \\
\hline & 2 & $(8.5,9,9.6)$ & $(9.6,9.8,10.3)$ & $(10.5,10.8,11)$ \\
\hline & 3 & $(4.2,5,5.5)$ & $(5.5,5.8,5.9)$ & $(6,6.2,6.6)$ \\
\hline \multirow{3}{*}{5} & 1 & $(3.8,4,4.2)$ & $(4.3,4.5,4.6)$ & $(4.65,4.8,5)$ \\
\hline & 2 & $(3.5,4,4.2)$ & $(4.4,4.5,4.7)$ & $(4.8,5,5.6)$ \\
\hline & 3 & $(9,10,10.5)$ & $(10.8,11,11.2)$ & $(11.3,11.5,11.6)$ \\
\hline
\end{tabular}

quality 700 and the minimum cost 6871.17 , and the corresponding optimal solution is reported in Table 6 . Table 6 shows the optimal supplier-level assignment mode and the order amounts of each product procured from its primary suppliers. For example, the primary suppliers for product 1 are the second, fourth, and fifth suppliers, and its level2 and level-3 suppliers are the first one and the third one, respectively. The order amounts from primary suppliers for product 1 are $76.5,75.5$, and 36.8 units.

The biobjective supplier selection model is solved several times through varying the target values $G_{1}$ and $G_{2}$ in order to obtain a suitable solution such that the decision maker can realize the trade-off of one criterion against another one. By computation, the relationship between quality and cost is shown in Figure 1. According to Figure 1, we can find

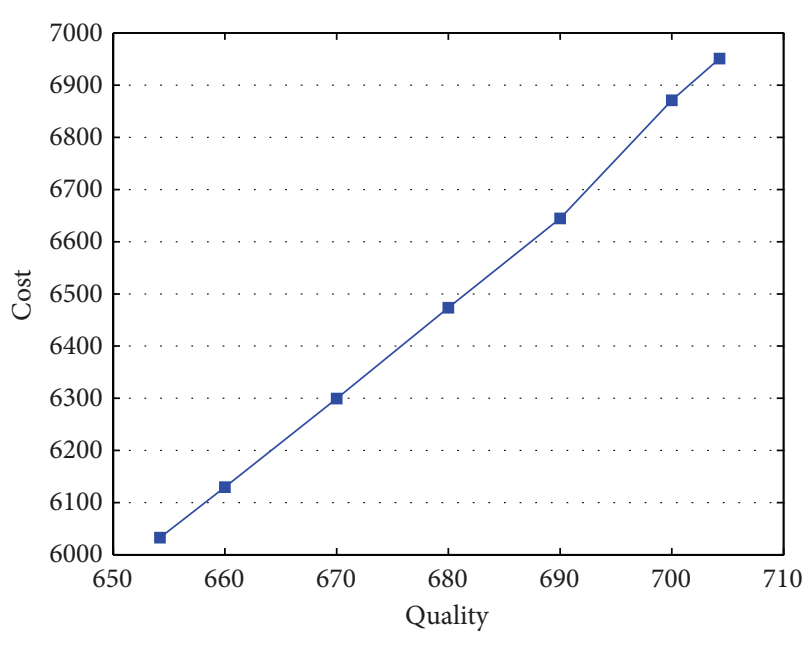

Figure 1: Relationship between quality and cost.

that increasing value of the first objective causes that of the second objective to increase. Also, decreasing value of the first objective causes that of the second objective to decrease. Therefore, it seems that, by raising the target value for any of the objectives, we can create more space for another objective to be improved. It is also concluded that there exist some positive correlations between the quality and the total cost.

In order to identify the impact of model parameters on solution quality, we try to look for the optimal solutions by adjusting independently the values of parameters $\alpha_{s p}$ and $\beta_{p}$. The computational results are reported in Table 7 , where the symbol $\left(\mathrm{obj}_{1}, \mathrm{obj}_{2}\right)$ represents the objective function values of corresponding optimal solution. From Table 7, we can find that the quality objective function value equals its target value 700 , while the total cost objective function value has a gap with its target value 6000 . On the one hand, the total cost increases gradually with respect to the increasing values of 
TABLE 5: Capacity data for supplier selection problem.

\begin{tabular}{lccccc}
\hline Product & & Capacity & \\
& Supplier 1 & Supplier 2 & Supplier 3 & Supplier 4 & Supplier 5 \\
\hline 1 & $(42,50,60)$ & $(75,90,100)$ & $(60,70,80)$ & $(75,80,90)$ & $(60,75,80)$ \\
2 & $(40,45,56)$ & $(80,100,115)$ & $(45,50,54)$ & $(180,200,250)$ & $(85,100,125)$ \\
3 & $(80,100,110)$ & $(16,20,30)$ & $(120,150,170)$ & $(40,50,65)$ & $(60,75,85)$ \\
\hline
\end{tabular}

TABLE 6: Solution result for biobjective supplier selection model.

\begin{tabular}{|c|c|c|c|c|c|c|c|}
\hline \multirow{2}{*}{ Supplier } & \multirow{2}{*}{ Product } & \multicolumn{3}{|c|}{ Supplier-level assignment } & \multicolumn{3}{|c|}{ Order amount } \\
\hline & & Level-1 & Level-2 & Level-3 & Level-1 & Level-2 & Level-3 \\
\hline \multirow{3}{*}{1} & 1 & 0 & 1 & 0 & 0 & 0 & 0 \\
\hline & 2 & 0 & 1 & 0 & 0 & 0 & 0 \\
\hline & 3 & 1 & 0 & 0 & 82 & 0 & 0 \\
\hline \multirow{3}{*}{2} & 1 & 1 & 0 & 0 & 76.5 & 0 & 0 \\
\hline & 2 & 1 & 0 & 0 & 82 & 0 & 0 \\
\hline & 3 & 0 & 1 & 0 & 0 & 0 & 0 \\
\hline \multirow{3}{*}{3} & 1 & 0 & 0 & 1 & 0 & 0 & 0 \\
\hline & 2 & 0 & 0 & 1 & 0 & 0 & 0 \\
\hline & 3 & 1 & 0 & 0 & 100.63 & 0 & 0 \\
\hline \multirow{3}{*}{4} & 1 & 1 & 0 & 0 & 75.5 & 0 & 0 \\
\hline & 2 & 1 & 0 & 0 & 145.23 & 0 & 0 \\
\hline & 3 & 0 & 0 & 1 & 0 & 0 & 0 \\
\hline \multirow{3}{*}{5} & 1 & 1 & 0 & 0 & 36.8 & 0 & 0 \\
\hline & 2 & 1 & 0 & 0 & 86.5 & 0 & 0 \\
\hline & 3 & 1 & 0 & 0 & 61.5 & 0 & 0 \\
\hline
\end{tabular}

TABLE 7: Parameters analysis about $\alpha_{s p}$ and $\beta_{p}$ for biobjective supplier selection model.

\begin{tabular}{lcccccc}
\hline \multicolumn{1}{c}{$\alpha_{s p}$} & $\beta_{p}=0.98$ & $\beta_{p}=0.9$ & $\beta_{p}=0.85$ & $\beta_{p}=0.8$ & $\beta_{p}=0.75$ & $\beta_{p}=0.7$ \\
\hline 0.95 & $(700,6859.32)$ & $(700,6839.59)$ & $(700,6819.84)$ & $(700,6800.10)$ & $(700,6784.85)$ & $(700,6778.12)$ \\
0.9 & $(700,6806.81)$ & $(700,6775.22)$ & $(700,6755.48)$ & $(700,6741.49)$ & $(700,6734.76)$ & $(700,6728.02)$ \\
0.7 & $(700,6565.37)$ & $(700,6554.59)$ & $(700,6547.85)$ & $(700,6541.12)$ & $(700,6534.37)$ & $(700,6527.27)$ \\
\hline
\end{tabular}

$\beta_{p}$ for the same parameter $\alpha_{s p}$. On the other hand, the total cost decreases gradually with respect to the decreasing values of $\alpha_{s p}$ for the same parameter $\beta_{p}$.

Lead time in supply chain management is a crucial parameter. To highlight the role of lead time in supplier selection planning, we perform some experiments to measure its effect. In this case, we report the computational results in Table 8 . Table 8 shows that there is much difference in terms of the optimal decision and corresponding objective value when the parameter $L$ changes. Furthermore, if the acceptable lead time turns small, the total cost has the increasing trend. So it is concluded that there exist some negative correlations between the total cost and the lead time.

\section{Conclusions}

In the present study, the fuzzy supplier selection problem is considered, including biobjective model, property analysis, solution method, and numerical experiments. The innovation results of the paper are summarized as follows.

(i) A biobjective fuzzy supplier selection model with uncertain variable cost, supplier capacity, and buyer demand is built, in which maximizing the total product quality and minimizing the total cost are our two objectives.

(ii) The basic properties of presented model are discussed (Theorems 1, 2, and 3). From the theoretical results, we obtain the analytical expressions of the expected value objective and credibility constraints which transform the original fuzzy model into its equivalent deterministic one.

(iii) A solution procedure integrated with the preemptive goal programming is designed, which transforms our model into a crisp single objective version. This transformation reduces computational complexity 
TABLE 8: Parameters analysis about $L$ for biobjective supplier selection model.

\begin{tabular}{|c|c|c|c|c|c|c|}
\hline \multirow{2}{*}{$L$} & \multirow{2}{*}{ Product } & \multicolumn{3}{|c|}{ Level } & \multirow{2}{*}{ Order amounts } & \multirow{2}{*}{$\left(\mathrm{obj}_{1}, \mathrm{obj}_{2}\right)$} \\
\hline & & 1 & 2 & 3 & & \\
\hline \multirow{3}{*}{2700} & 1 & $\left(s_{2}, s_{4}, s_{5}\right)$ & $s_{1}$ & $s_{3}$ & $(76.5,75.5,35)$ & \multirow{3}{*}{$(681,6725.43)$} \\
\hline & 2 & $\left(s_{2}, s_{4}, s_{5}\right)$ & $s_{1}$ & $s_{3}$ & $(82,131.53,86.5)$ & \\
\hline & 3 & $\left(s_{1}, s_{3}, s_{5}\right)$ & $s_{2}$ & $s_{4}$ & $(82,95.5,61.5)$ & \\
\hline \multirow{3}{*}{2800} & 1 & $\left(s_{2}, s_{4}, s_{5}\right)$ & $s_{1}$ & $s_{3}$ & $(76.5,75.5,36.8)$ & \multirow{3}{*}{$(700,6871.17)$} \\
\hline & 2 & $\left(s_{2}, s_{4}, s_{5}\right)$ & $s_{1}$ & $s_{3}$ & $(82,145.23,86.5)$ & \\
\hline & 3 & $\left(s_{1}, s_{3}, s_{5}\right)$ & $s_{2}$ & $s_{4}$ & $(82,100.63,61.5)$ & \\
\hline \multirow{3}{*}{2900} & 1 & $\left(s_{2}, s_{4}, s_{5}\right)$ & $s_{1}$ & $s_{3}$ & $(59.37,75.5,52.13)$ & \multirow{3}{*}{$(700,6644.16)$} \\
\hline & 2 & $\left(s_{2}, s_{4}, s_{5}\right)$ & $s_{1}$ & $s_{3}$ & $(82,154.93,86.5)$ & \\
\hline & 3 & $\left(s_{1}, s_{3}, s_{5}\right)$ & $s_{2}$ & $s_{4}$ & $(82,123,34)$ & \\
\hline \multirow{3}{*}{3000} & 1 & $\left(s_{2}, s_{4}, s_{5}\right)$ & $s_{1}$ & $s_{3}$ & $(56.52,75.5,54.98)$ & \multirow{3}{*}{$(700,6470.64)$} \\
\hline & 2 & $\left(s_{2}, s_{4}, s_{5}\right)$ & $s_{1}$ & $s_{3}$ & $(82,150.79,86.5)$ & \\
\hline & 3 & $\left(s_{1}, s_{3}, s_{4}\right)$ & $s_{5}$ & $s_{2}$ & $(82,123,41)$ & \\
\hline \multirow{3}{*}{3100} & 1 & $\left(s_{3}, s_{4}, s_{5}\right)$ & $s_{1}$ & $s_{2}$ & $(52.49,73,61.5)$ & \multirow{3}{*}{$(700,6400.62)$} \\
\hline & 2 & $\left(s_{2}, s_{4}, s_{5}\right)$ & $s_{1}$ & $s_{3}$ & $(82,158.06,86.5)$ & \\
\hline & 3 & $\left(s_{1}, s_{3}, s_{5}\right)$ & $s_{2}$ & $s_{4}$ & $(82,123,34)$ & \\
\hline
\end{tabular}

and makes the application of our procedure more understandable.

(iv) A numerical example is performed to verify the effectiveness of the proposed model for illustration purpose. The computational results verify that our solution method works well and provides acceptable solution, which hopefully can help decision maker in management.

\section{Notations}

$S: \quad$ Set of suppliers considered, indexed by

$s, s=1,2, \ldots, n$

$P: \quad$ Set of products, indexed by

$p, p=1,2, \ldots,|P|$

$R: \quad$ Set of levels, indexed by $r, r=1,2, \ldots, n-m+1$

$F_{r s p}$ : The fixed cost that supplier $s$ charges for product $p$ as level- $r$ supplier

$Q_{s p}$ : The quality level of unit product $p$ from its supplier $s$

$L_{r s p}$ : The lead time for unit product $p$ from its level- $r$ supplier $s$

$L: \quad$ The maximal lead time

$\xi_{r s p}$ : The fuzzy cost for unit product $p$ from its level- $r$ supplier $s$

$\eta_{s p}$ : The fuzzy capacity at supplier $s$ for product $p$

$\zeta_{p}: \quad$ The fuzzy demand for product $p$

$x_{r s p}$ : A binary variable indicating whether supplier $s$ is assigned as a level- $r$ supplier for product $p$ or not

$y_{1 s p}:$ The order of product $p$ distributed from its primary supplier $s$.

\section{Conflict of Interests}

The author declares that there is no conflict of interests regarding the publication of this paper.

\section{Acknowledgment}

This work is supported partially by the National Natural Science Foundation of China (no. 61374184).

\section{References}

[1] R. U. Bilsel and A. Ravindran, "A multiobjective chance constrained programming model for supplier selection under uncertainty," Transportation Researcht B: Methodological, vol. 45, no. 8, pp. 1284-1300, 2011.

[2] B. Balcik and D. Ak, "Supplier selection for framework agreements in humanitarian relief," Production and Operations Management, vol. 23, no. 6, pp. 1028-1041, 2014.

[3] A. Federgruen and N. Yang, "Selecting a portfolio of suppliers under demand and supply risks," Operations Research, vol. 56, no. 4, pp. 916-936, 2008.

[4] S.-M. Guu, M. Mehlawat, and S. Kumar, "A multiobjective optimization framework for optimal selection of supplier portfolio," Optimization, vol. 63, no. 10, pp. 1491-1512, 2014.

[5] A. Hosseininasab and A. Ahmadi, "Selecting a supplier portfolio with value, development, and risk consideration," European Journal of Operational Research, vol. 245, no. 1, pp. 146-156, 2015.

[6] B. Hu and D. Kostamis, "Managing supply disruptions when sourcing from reliable and unreliable suppliers," Production and Operations Management, vol. 24, no. 5, pp. 808-820, 2015.

[7] T. Sawik, "Joint supplier selection and scheduling of customer orders under disruption risks: single vs. dual sourcing," Omega, vol. 43, pp. 83-95, 2014. 
[8] L. A. Zadeh, "Fuzzy sets," Information and Control, vol. 8, no. 3, pp. 338-353, 1965.

[9] B. Liu and Y.-K. Liu, "Expected value of fuzzy variable and fuzzy expected value models," IEEE Transactions on Fuzzy Systems, vol. 10, no. 4, pp. 445-450, 2002.

[10] Y. Liu and X. Bai, "Studying interconnections between two classes of two-stage fuzzy optimization problems," Soft Computing, vol. 17, no. 4, pp. 569-578, 2013.

[11] X. J. Bai and Y. K. Liu, "Semideviations of reduced fuzzy variables: a possibility approach," Fuzzy Optimization and Decision Making, vol. 13, no. 2, pp. 173-196, 2014.

[12] A. Amid, S. H. Ghodsypour, and C. O’Brien, "A weighted maxmin model for fuzzy multi-objective supplier selection in a supply chain," International Journal of Production Economics, vol. 131, no. 1, pp. 139-145, 2011.

[13] S. M. Arabzad, M. Ghorbani, J. Razmi, and H. Shirouyehzad, "Employing fuzzy TOPSIS and SWOT for supplier selection and order allocation problem," International Journal of Advanced Manufacturing Technology, vol. 76, no. 5-8, pp. 803-818, 2014.

[14] F. Arikan, "An interactive solution approach for multiple objective supplier selection problem with fuzzy parameters," Journal of Intelligent Manufacturing, vol. 26, no. 5, pp. 989-998, 2015.

[15] A. H. Azadnia, M. Z. M. Saman, and K. Y. Wong, "Sustainable supplier selection and order lot-sizing: an integrated multiobjective decision-making process," International Journal of Production Research, vol. 53, no. 2, pp. 383-408, 2015.

[16] A. F. Guneri, A. Yucel, and G. Ayyildiz, "An integrated fuzzylp approach for a supplier selection problem in supply chain management," Expert Systems with Applications, vol. 36, no. 5, pp. 9223-9228, 2009.

[17] W. Pan, F. Wang, Y. Guo, and S. Liu, "A fuzzy multiobjective model for supplier selection under considering stochastic demand in a supply chain," Mathematical Problems in Engineering, vol. 2015, Article ID 174585, 8 pages, 2015.

[18] J. Chai, J. N. K. Liu, and E. W. T. Ngai, "Application of decisionmaking techniques in supplier selection: a systematic review of literature," Expert Systems with Applications, vol. 40, no. 10, pp. 3872-3885, 2013.

[19] L. V. Snyder and M. S. Daskin, "Reliability models for facility location: the expected failure cost case," Transportation Science, vol. 39, no. 3, pp. 400-416, 2005.

[20] H. S. Wang, C. H. Tu, and K. H. Chen, "Supplier selection and production planning by using guided genetic algorithm and dynamic nondominated sorting genetic algorithm II approaches," Mathematical Problems in Engineering, vol. 2015, Article ID 260205, 15 pages, 2015.

[21] A. Chinchuluun and P. M. Pardalos, "A survey of recent developments in multiobjective optimization," Annals of Operations Research, vol. 154, no. 1, pp. 29-50, 2007.

[22] Y. Collette and P. Siarry, Multiobjective Optimization: Principles and Case Studies, Springer, Berlin, Germany, 2003.

[23] K. Deb, Multi-Objective Optimization Using Evolutionary Algorithms, Wiley, New York, NY, USA, 2001.

[24] A. Charnes, W. W. Cooper, and R. O. Ferguson, "Optimal estimation of executive compensation by linear programming," Management Science, vol. 1, no. 2, pp. 138-151, 1955.

[25] A. Charnes and W. W. Cooper, Management Models and Industrial Applications of Linear Programming, Wiley, New York, NY, USA, 1961.

[26] Y. Ijiri, Management Goals and Accounting for Control, North Holland Publishing, Amsterdam, The Netherlands, 1965.
[27] S. M. Lee, Goal Programming for Decision Analysis, Auerbach, Philadelphia, Pa, USA, 1972. 


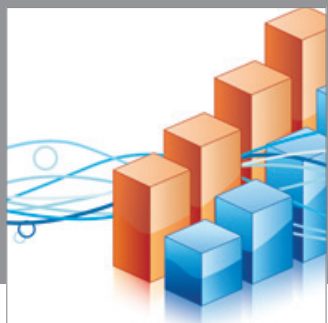

Advances in

Operations Research

mansans

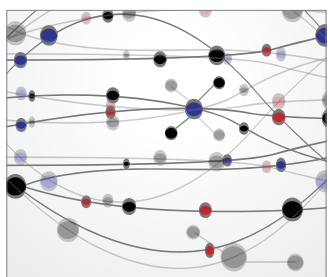

The Scientific World Journal
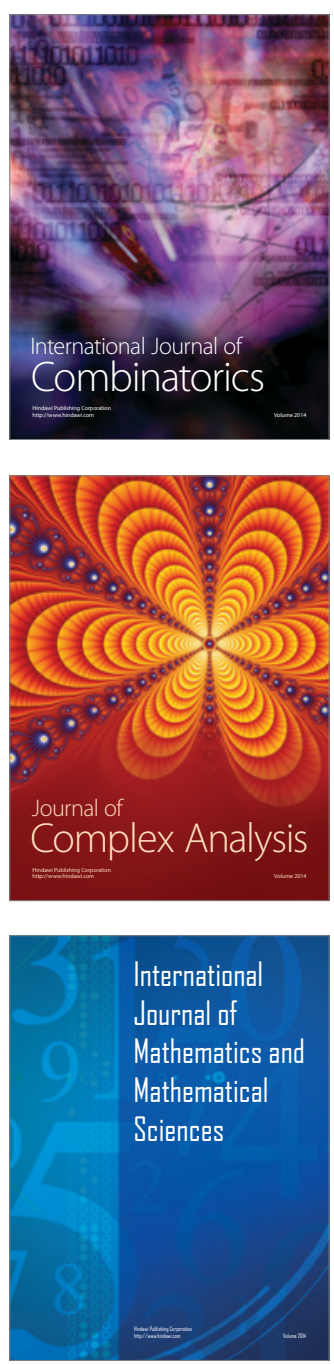
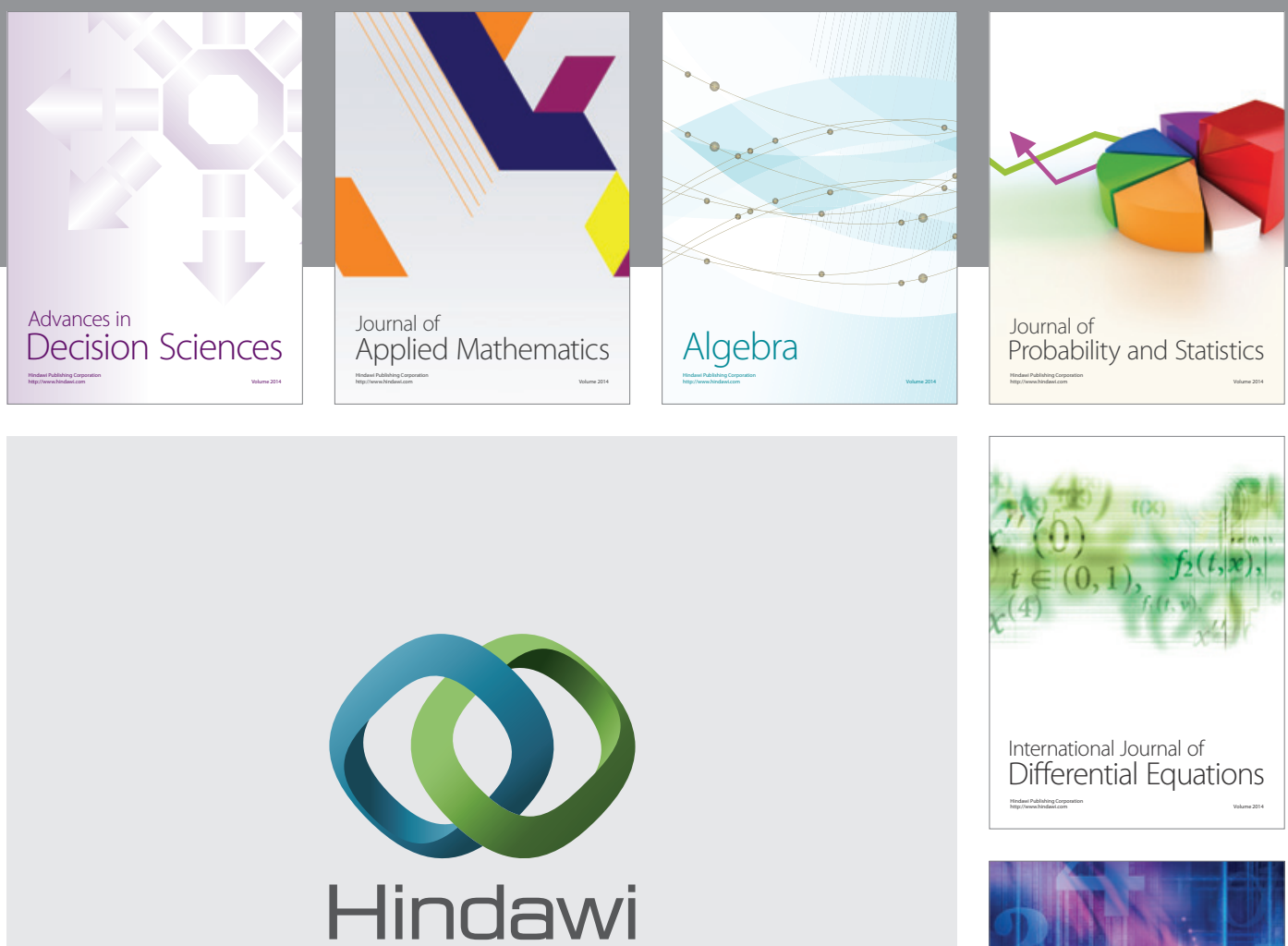

Submit your manuscripts at http://www.hindawi.com
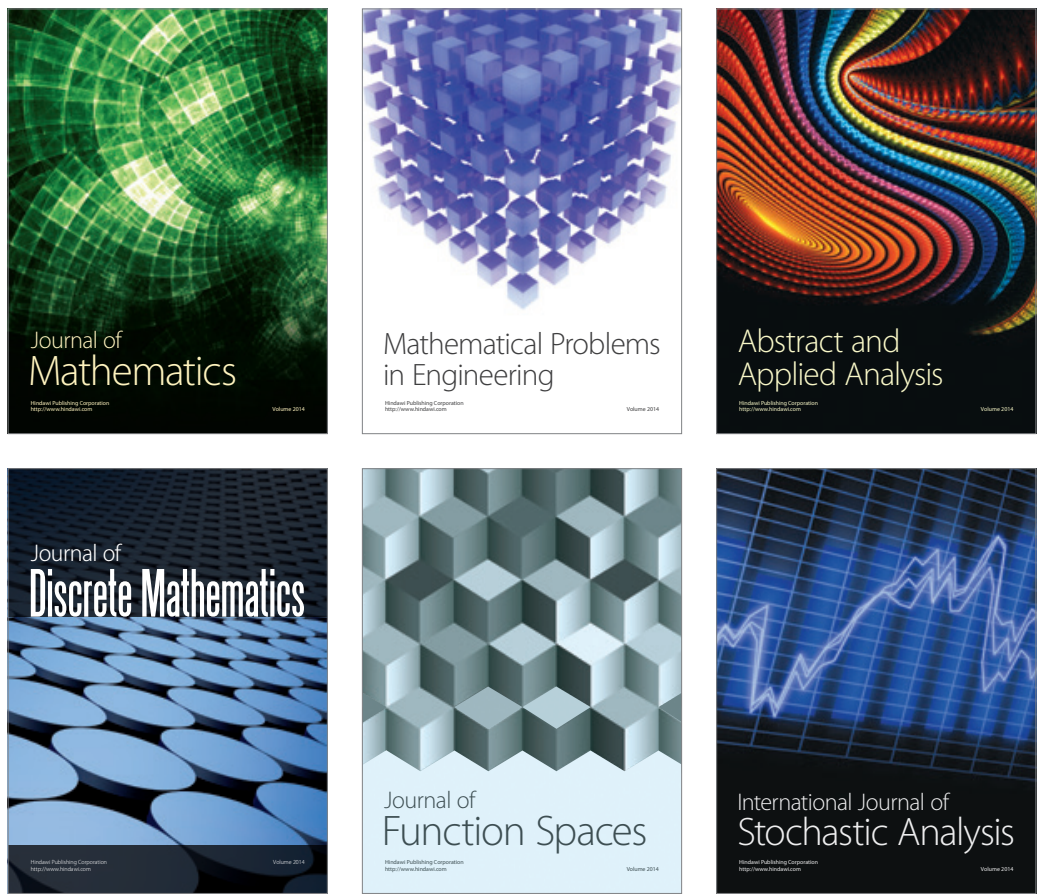

Journal of

Function Spaces

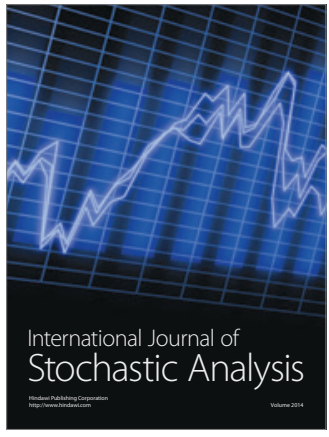

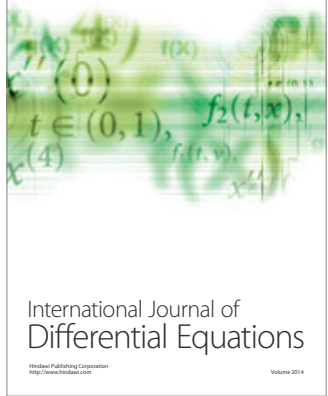
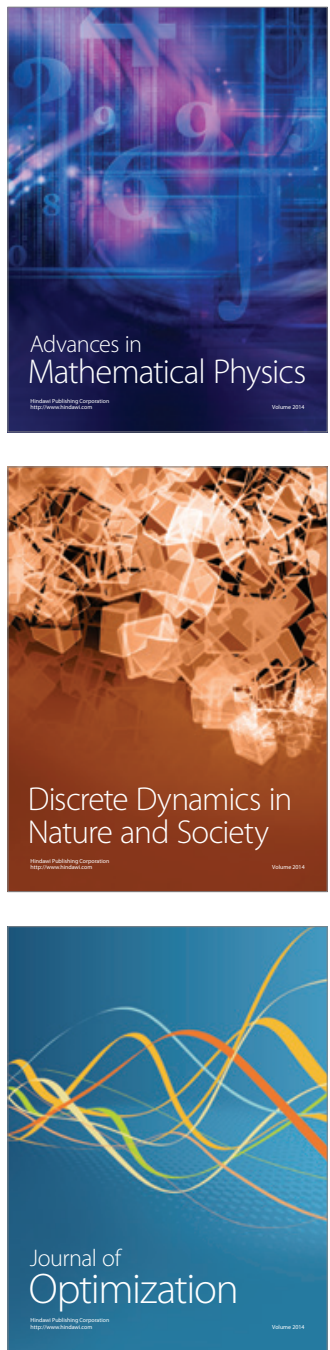